\title{
DISEÑO DE UN PLAN DE INTERVENCIÓN PARA LA PARTICIPACIÓN DE LOS PADRES EN EL PROCESO EDUCATIVO DE EDUCANDOS CON TRASTORNO POR DÉFICIT DE ATENCIÓN Y COMPORTAMIENTO PERTURBADOR
}

\author{
DESIGN OF AN INTERVENTION PLAN FOR THE PARTICIPATION OF \\ PARENTS IN THE EDUCATIONAL PROCESS OF STUDENTS WITH \\ ATTENTION DEFICIT DISORDER AND DISTURBING BEHAVIOR
}

\section{Luis Enrique Vásquez Gimenez ${ }^{1}$}

\section{RESUMEN}

El propósito de la siguiente investigación es el diseño de un Plan de Intervención de los padres en el Proceso Educativo de Educandos con Trastorno por Déficit de Atención y comportamiento perturbador que acuden al Núcleo Psicoeducativo de la Unidad Educativa Municipal Simón Rodríguez. El niño con este trastorno se torna impulsivo y distraído, trastorno que interfiere en la capacidad para concentrarse; en él existen áreas que afectan su adaptación en cuanto a cómo seguir instrucciones, organizarse, solucionar problemas y relacionarse con los demás. La investigación fue un proyecto factible, apoyado en una investigación de campo con carácter descriptivo. Se seleccionó el cuestionario tipo encuesta con preguntas cerradas y la observación directa con el fin de examinar el grado de información que manejan los padres sobre el TDAH. La población es censal, conformada por 6 padres de los educandos que acuden al Núcleo Psicoeducativo de la U.E.M. Simón Rodríguez, correspondientes a la primera etapa de educación Básica, diagnosticados por el especialista en la materia, y atendidos por el Núcleo Psicoeducativo en el turno de la tarde. Para el desarrollo de la investigación fue necesario identificar el nivel de información que poseen los padres para participar de manera efec-

1 Aspirante al grado de Doctor en Gestión y Ciencias de la Educación. Magíster en Educación Mención Orientación. Licenciado en Filosofía y Educación Integral. Docente e Investigador de la Red Laureate Internacional Universities (UPN-Lima).Email: tobit7@hotmail.com 
tiva en el proceso terapéutico de sus hijos con TDAH. Luego, diagnosticar las necesidades de atención de los padres para abordar las conductas que presentan los educandos con TDAH y sus necesidades. Y por último, el Diseño de un Plan de Intervención para la Participación de los Padres en el Proceso Educativo sus hijos con Trastorno por Déficit de Atención y Comportamiento Perturbador.

Palabras ClaVe: Trastorno por Déficit de Atención. Padres. Plan. Intervención. Estrategia.

\section{ABSTRACT}

The purpose of the following research is the design of a Parent Intervention Plan in the Educational Process of Educandos with Attention Deficit Disorder and disturbing behavior that come to the Psychoeducational Nucleus of the Simón Rodríguez Municipal Educational Unit. The child with this disorder becomes impulsive and distracted, a disorder that interferes with the ability to concentrate; There are areas that affect your adaptation in how to follow instructions, organize, solve problems and relate to others. The research was a feasible project, supported by a descriptive field research. The questionnaire type survey with closed questions and direct observation was selected in order to examine the degree of information that the parents handle about ADHD. The population is census, made up of 6 parents of students who attend the Psychoeducational Nucleus of the U.E.M. Simón Rodríguez, corresponding to the first stage of basic education, diagnosed by the specialist in the subject, and attended by the Psychoeducational Nucleus in the afternoon shift. For the development of the research was necessary to identify the level of information that parents have to participate effectively in the therapeutic process of their children with ADHD. Then, diagnose the needs of parents to address the behaviors that students with ADHD and their needs. And finally, the Design of an Intervention Plan for the Participation of Parents in the Educational Process their children with Attention Deficit Disorder and Disturbing Behavior.

KEY WORDS: Attention Deficit Disorder. Parents. Plan. Intervention. Strategy.

\section{INTRODUCCIÓN}

La integración social de las personas con necesidades educativas especiales es susceptible a diferentes factores desde el mundo familiar compuesto por los padres, así como el escolar, legal, laboral, etc. Sin embargo, uno de los grandes problemas a los que se enfrenta el especialista cuando trata a estos pacientes es el convencer a los familiares que ellos también deben ser incluidos en el tratamiento. Según Marcelli (1996), con mucha frecuencia en estos hogares se observan inadecuadas reglas de disciplina, mucha flexibilidad en cuanto a la fijación de límites, discusiones y desautorizaciones frecuentes entre los miembros.

La presente investigación plantea un tema que hoy día es sumamente estudiado, desde diferentes enfoques, se trata del Trastorno por Déficit de Atención y 
comportamiento perturbador (TDAH), el cual consiste en un síndrome que afecta significativamente la vida de las personas. Se caracteriza por una dificultad o incapacidad para mantener la atención voluntaria frente a determinadas actividades tanto en el ámbito académico, como cotidiano. Por ende, las relaciones sociales se ven afectadas por el hecho que es muy difícil para una persona de estas características seguir reglas o normas rígidas de comportamiento. Habitualmente, se asocia este déficit a una falta de autocontrol y sobretodo dificultades en la toma de conciencia de los errores propios para la persona afectada.

En cuanto al tratamiento existen diferentes posturas, las que promueven el uso de fármacos y las que prefieren abordar el problema a través del desarrollo de estrategias de trabajo en el ámbito escolar, social y familiar. Precisamente dentro del contexto familiar se basará el estudio en cuanto a la participación de los padres en el tratamiento de sus hijos con TDAH debido a que el trabajo que se haga con estos representa un elemento fundamental para el éxito de la atención especializada, por esta razón se les debe considerar, así como de mantenerlos informados y comprometidos siempre en las labores a realizar con sus hijos.

\section{PROBLEMA}

¿Cuál es el nivel de información que poseen los padres de niños diagnosticados con TDAH y qué aspectos se deben considerar para apoyarlos en el proceso educativo?

\section{OBJETIVO GENERAL}

Diseñar un Plan de Intervención para la participación de los padres en el Proceso Educativo de educandos con Trastorno por Déficit de Atención y Comportamiento Perturbador.

\section{OBJETIVOS ESPECÍFICOS}

1. Diagnosticar el nivel de información que poseen los padres de educandos con Trastorno por Déficit de atención y Comportamiento Perturbador, del Núcleo Psicoeducativo de la UEM Simón Rodríguez, en el turno de la tarde, para participar de manera efectiva en el proceso terapéutico de sus hijos.

2. Analizar los planteamientos teóricos relacionados con el Trastorno por Déficit de Atención y Comportamiento Perturbador.

3. Describir los aspectos a considerar en la elaboración de un plan de intervención para padres de educandos con Trastorno por Déficit de Atención y Comportamiento Perturbador. 
4. Diseñar un Plan de Intervención para la participación de los padres en el Proceso Educativo de educandos con Trastorno por Déficit de Atención y Comportamiento Perturbador, para el fortalecimiento y desarrollo de sus habilidades emocionales, sociales y cognitivas.

\section{MATERIAL Y MÉTODOS}

La investigación realizada se puede clasificar como un proyecto factible apoyado en una investigación de campo, ya que el análisis sistemático de problemas fue con el propósito de describirlos, explicar sus causas y efectos, entender su naturaleza y factores constituyentes, el cual según Silva (2006), "es el medio por el cual se lleva a cabo la investigación”. Está apoyada en una investigación de campo la cual es definida como "investigaciones que se realizan en el medio donde se desarrolla el problema, o en el lugar donde se encuentra el objeto de estudio: el investigador recoge la información directamente de la realidad" (ibídem p. 35).

La misma se encuentra enmarcada en un estudio de tipo descriptivo, ya que se describen los hechos tal como se dan, según afirma Tamayo (1994), la investigación descriptiva comprende la descripción, registro, análisis e interpretación de la naturaleza actual composición o proceso de fenómenos. El enfoque se hace sobre conclusiones dominantes o sobre como una persona, grupo o cosa se conduce o funciona en el presente. La investigación descriptiva trabaja sobre realidades de hechos y su característica fundamental, es la de presentar una interpretación correcta. (p. 32).

El nivel de investigación se refiere al grado de profundidad con que se aborda un objeto o fenómeno. Con lo que se concluye que el trabajo es de nivel descriptivo. Ya que "se describen los hechos tal como se dan por lo que se efectúa sobre un objeto o tema conocido o estudiado, y sus resultados constituyen una visión aproximada de dicho objeto”. (Arias, 2006) (p. 24).

La población según Arias (2006) "es un conjunto finito o infinito de elementos con características comunes para los cuales serán extensivas las conclusiones de la investigación. Está delimitada por el problema y por los objetivos del estudio" (p. 28). En la actual investigación se trabajará con una población censal, es decir, con los seis padres de los niños diagnosticados con trastorno por déficit de atención y comportamiento perturbador que acuden al núcleo psicoeducativo de la UEM Simón Rodríguez en el turno de la tarde. Tamayo (1994), nos refiere que una población está determinada por sus características definitorias, por tan- 
to, el conjunto de elementos que posea esta característica se denomina población o universo.

La Técnica según Arias (2006) es "el procedimiento o forma particular de obtener datos o información”. En este caso se utilizará la Técnica de la Observación también conceptualizada como "una técnica que pretende obtener información que suministra un grupo o muestra de sujetos acerca de sí mismos, o en relación con un tema en particular". (p. 34)

Por otra parte, según Ramírez (2005) el instrumento de recolección de datos es "cualquier recurso, dispositivo o formato (en papel o digital), que se utiliza para obtener, registrar o almacenar la información”. El instrumento utilizado en esta investigación es el cuestionario definido como "la modalidad de encuesta que se realiza de forma escrita mediante un instrumento o formato en papel contentivo de una serie de preguntas". Dicho cuestionario es de preguntas cerradas "son aquellas que establece previamente las opciones de respuestas que puede elegir el encuestado". (Idem) Para el avance de la presente investigación se desarrolló un cuestionario con doce ítems, con respuestas cerradas, con los cuales se pretendía valorar en primer lugar la participación de los padres con respecto al Trastorno por Déficit de Atención y Comportamiento Perturbador que presentan sus hijos; y por otro lado conocer las conductas que presentan estos niños ya diagnosticados y su interrelación social en todos los ámbitos de su vida.

Para Arias (2006), "el cuestionario es un instrumento que se utiliza para obtener opiniones, conocer actitudes, donde las interrogantes precedentes y las que piense el investigador van a posibilitar reconstruir la historia. Es importante que los investigadores pregunten, averigüen, cuestionen, reflexionen, analicen y escriban sobre lo indagado". (p. 37)

Cabe resaltar que el instrumento de recolección de datos fue revisado por 3 especialistas tales como: psicólogo clínico, docente especialista y asesor metodológico. Para luego ser aplicado a los seis padres de los niños que asisten al núcleo psicoeducativo de la UEM Simón Rodríguez, en el turno de la tarde, que han sido diagnosticados con este Trastorno por el Especialista en la materia.

\section{RESULTADOS}

Los resultados obtenidos en la investigación, sobre la base de los instrumentos aplicados al estudio de caso. De resultados numéricos obtenidos se transformaron en resultados porcentuales para poderlos comparar. Estos se analizan por 
separado y se presentaran en gráficos en los que se establecerá un contraste de los mismos para determinar puntos convergentes y divergentes entre ambos. Luego se refuerza el producto del contraste con las bases teóricas para dar mayor objetividad a los resultados y permitir así el desarrollo del plan de intervención como propuesta de esta investigación a fin de mejorar el problema planteado en este estudio.

Una vez tabulada la información obtenida a partir de la aplicación de los instrumentos de recolección de datos, se procedió a utilizar la técnica estadística para comprender e interpretar los resultados y así dar respuesta a las interrogantes planteadas. En tal sentido, se ha utilizado el Análisis descriptivo-cuantitativo, a partir del cual se resumen los datos y características obtenidos en forma porcentual, distribuidos y agrupados en clases, con el fin de observar el comportamiento de cada variable en la población. Asimismo, se considera cuantitativo porque el análisis de la información se efectúa con todos los datos en forma numérica (Sabino, 1992).

Los resultados obtenidos en la investigación se presentan en cuadros estadísticos que contienen una distribución absoluta y porcentual de frecuencia. Cada uno está organizado en tres (3) columnas, en la primera aparecen los ítem, en la segunda la frecuencia absoluta y en la tercera la frecuencia relativa o porcentual. La interpretación de cada ítem se realizó inmediatamente después de su presentación. Seguidamente, se presentan los resultados de la encuesta aplicada a los individuos de la comunidad o institución seleccionada, como se aprecia a continuación.

Tabla 1

\section{EL COMPORTAMIENTO}

Ítem $\mathrm{N}^{\circ}$ 1: iAcude usted a la escuela solicitando asesoramiento o ayuda ante el comportamiento incontrolable de su hijo?

\begin{tabular}{ccc}
\hline Ítem 1 & Frecuencia & Porcentaje \\
\hline $\mathrm{Si}$ & 3 & $50 \%$ \\
$\mathrm{No}$ & 3 & $50 \%$ \\
\hline Total & 6 & $100 \%$ \\
\hline
\end{tabular}


Tabla 2

ASISTENCIA ESPECIALIZADA

Ítem $\mathrm{N}^{\circ}$ 2: ¿Su hijo acude al núcleo psicoeducativo, terapista del lenguaje u otro especialista?

\begin{tabular}{ccc}
\hline Ítem 2 & Frecuencia & Porcentaje \\
\hline $\mathrm{Si}$ & 6 & $100 \%$ \\
$\mathrm{No}$ & 0 & $0 \%$ \\
\hline Total & 6 & $100 \%$ \\
\hline
\end{tabular}

Tabla 3

TRATAMIENTO FARMACOLÓGICO

Ítem $\mathrm{N}^{\circ}$ 3: iInforma al maestro(a) que su hijo(a) está recibiendo tratamiento farmacológico?

\begin{tabular}{ccc}
\hline Item 3 & Frecuencia & Porcentaje \\
\hline $\mathrm{Si}$ & 3 & $50 \%$ \\
$\mathrm{No}$ & 3 & $50 \%$ \\
\hline Total & 6 & $100 \%$ \\
\hline
\end{tabular}

Tabla 4

COMPORTAMIENTO

Ítem $\mathrm{N}^{\circ}$ 4: ¿Comunica sus dificultades para controlar el comportamiento de su hijo(a) en el hogar?

\begin{tabular}{ccc}
\hline Ítem 4 & Frecuencia & Porcentaje \\
\hline $\mathrm{Si}$ & 5 & $83 \%$ \\
$\mathrm{No}$ & 1 & $17 \%$ \\
\hline Total & 6 & $100 \%$ \\
\hline
\end{tabular}


De acuerdo a la tabla 1, de los 6 padres consultados, sólo 3 respondieron que sí acuden a la escuela para consultar y solicitar asesoramiento sobre el comportamiento incontrolable de su hijo lo que corresponde al 50\% de la población; el otro 50\% no. El porcentaje de requerimiento de ayuda solicitado a la escuela es equilibrado, hoy día el papel de la escuela es esencial en lo que se refiere a situaciones especiales, de allí la importancia de la comunicación entre padres y maestros. Tal como se plantea en el sistema educativo que manifiesta que la escuela es concebida como el centro de formación del individuo que la sociedad necesita. (París, 2003).

Como se puede notar en la tabla 2, el 100\% de los hijos reciben atención especializada. Esto indica que los padres están informados sobre la atención especializada que ofrece la escuela a sus hijos. Situación que representa una fortaleza debido a que el abordaje del TDAH es a través del equipo multidisciplinario. En este sentido se plantea que dentro de la línea de trabajo juega un rol muy importante el psicólogo clínico y educacional, el docente especialista en dificultades de aprendizaje, los docentes regulares de los niños y especialmente los padres. (Ross, 1997).

De acuerdo a la tabla 3 se evidencia que el $50 \%$ de los padres informa al maestro que su hijo está recibiendo tratamiento farmacológico, el otro 50\% no. Llama la atención que un porcentaje considerable de padres no informen al docente el tratamiento farmacológico que recibe su hijo. La importancia del rol del docente no debe ser subestimada, este debe estar al tanto de todo el cuadro clínico de su alumno. Un tratamiento es exitoso si participan en cooperación docentes, especialistas y sin duda los padres. Sin embargo hay que tomar en cuenta los prejuicios generados por la medicación. En este sentido Ross (1997) plantea "Existe una postura antagónica de tratamiento y manejo de niños con Trastorno por Déficit de Atención y comportamiento Perturbador, la cual presenta alternativas que dejan fuera los fármacos".

Se observa en la tabla 4 que el $83 \%$ de los padres sí comunica las dificultades para controlar el comportamiento de sus hijos y el otro 17\% no. En esta situación se evidencia un porcentaje respetable de padres que manifiestan sus necesidades en la dificultad que presentan en el control del comportamiento del hijo con TDAH. Una de las más grandes dificultades entre otras que enfrentan los padres es la manera de manejar el comportamiento ocasionado por el TDAH en sus hijos, debido a la cantidad de conflictos que surgen entre los familiares y el caos que se genera en el hogar. Sin embargo, en la escuela el porcentaje de comunicación en este sentido es menor. En cuanto al comportamiento Marcelli (1996) plantea "es muy difícil para una persona con estas características seguir reglas o normas rígidas de comportamiento". 
Tabla 5

ATENCIÓN Y OLVIDOS

Ítem $\mathrm{N}^{\circ}$ 5: ¿Consulta sobre qué hacer ante los problemas de desatención, olvidos, errores de su hijo en el hogar?

\begin{tabular}{ccc}
\hline Ítem 5 & Frecuencia & Porcentaje \\
\hline $\mathrm{Si}$ & 3 & $50 \%$ \\
$\mathrm{No}$ & 3 & $50 \%$ \\
\hline Total & 6 & $100 \%$ \\
\hline
\end{tabular}

Tabla 6

INTEGRACIÓN SOCIAL

Ítem $\mathrm{N}^{\circ}$ 6: ¿Consulta sobre las dificultades de integración y relación social de su hijo(a), aislamiento, rechazo, conflictos, entre otros?

\begin{tabular}{ccc}
\hline Ítem 6 & Frecuencia & Porcentaje \\
\hline Si & 4 & $67 \%$ \\
No & 2 & $33 \%$ \\
\hline Total & 6 & $100 \%$ \\
\hline
\end{tabular}

Tabla 7

AYUDA PSICOLÓGICA PARA LOS PADRES

Ítem $\mathrm{N}^{\circ} 7$ : $i$ Tiene necesidad de ayuda psicológica profesional ya sea por ansiedad y depresión ante el comportamiento de su hijo?

\begin{tabular}{ccc}
\hline Ítem 7 & Frecuencia & Porcentaje \\
\hline Si & 2 & $33 \%$ \\
No & 4 & $67 \%$ \\
\hline Total & 6 & $100 \%$ \\
\hline
\end{tabular}


Como se puede observar en los resultados de la tabla 5, el 50\% de los padres consultan qué hacer ante los problemas de desatención, olvido, errores de su hijo(a) en el hogar, y el otro 50\% no. Los niños con TDAH, son olvidadizos y no prestan atención a los detalles por lo que siempre se enfrentan a situaciones de pérdida de objetos y posibles accidentes domésticos que ocasiona en los padres situaciones estresantes. En este sentido encontramos que según el DSM-IV-TR Brevario (2002) "el rasgo más relevante o más decisivo de la existencia de un posible déficit atencional es la falta de atención o inatención".

De acuerdo a la tabla 6, el 67\% de los padres si consultan sobre la integración y relación social de los hijos el 33\% no. Los síntomas por TDAH, crean conflictos en las relaciones personales y familiares, es por ello que se les dificulta actuar adecuadamente en casa o en sitios públicos. Se evidencia esta problemática que plantean los padres de allí la preocupación por parte de estos en buscar información debido a las consecuencias a nivel personal, familiar y social de esta situación. Con relación a lo anterior Van-Wielink, (2004), manifiesta que las relaciones sociales son una habilidad poco comprendida y muy importante para el sano desempeño de la personalidad del niño.

Se pudo comprobar a través de la tabla 7 que el $67 \%$ expresó no tener necesidad de ayuda psicológica profesional ante el comportamiento de su hijo causado por el TDAH. El otro 33\% sí. Esta situación representa una fortaleza ya que es importante que los padres estén emocional y físicamente sanos dada la responsabilidad como representantes de un niño con TDAH. Tal como lo dice Marcelli (1996) "Los padres representan la fuente de seguridad, los modelos a seguir, el reflejo mismo de lo que los niños sienten y son".

Tabla 8

PROBLEMAS DE APRENDIZAJE

Ítem $\mathrm{N}^{\circ}$ 8: iSabe qué hacer ante las dificultades de su hijo(a) para mantener la atención en tareas o en actividades de aprendizaje?

\begin{tabular}{ccc}
\hline Ítem 8 & Frecuencia & Porcentaje \\
\hline $\mathrm{Si}$ & 1 & $17 \%$ \\
$\mathrm{No}$ & 5 & $83 \%$ \\
\hline Total & 6 & $100 \%$ \\
\hline
\end{tabular}


Tabla 9

\section{SEGUIMIENTO DE INSTRUCCIONES}

Ítem $\mathrm{N}^{\circ}$ 9: ¿Solicita información para saber qué hacer cuando su hijo(a) no sigue instrucciones y no finaliza las tareas escolares, encargos y deberes escolares?

\begin{tabular}{ccc}
\hline Ítem 9 & Frecuencia & Porcentaje \\
\hline $\mathrm{Si}$ & 3 & $50 \%$ \\
$\mathrm{No}$ & 3 & $50 \%$ \\
\hline Total & 6 & $100 \%$ \\
\hline
\end{tabular}

Tabla 10

ORGANIZACIÓN DE ACTIVIDADES

Ítem $\mathrm{N}^{\circ}$ 10: ¿sabe qué hacer cuando su hijo(a) tiene dificultades para organizar tareas y actividades?

\begin{tabular}{ccc}
\hline Ítem 10 & Frecuencia & Porcentaje \\
\hline $\mathrm{Si}$ & 1 & $17 \%$ \\
$\mathrm{No}$ & 5 & $83 \%$ \\
\hline Total & 6 & $100 \%$ \\
\hline
\end{tabular}

Tabla 11

PÉRDIDA DE OBJETOS

Ítem $\mathrm{N}^{\circ}$ 11: ¿Sabe cómo ayudar a su hijo(a) cuando pierde objetos necesarios para tareas o actividades? Ej. Juguetes, material escolar, lápices, libros o herramientas)

\begin{tabular}{ccc}
\hline Ítem 11 & Frecuencia & Porcentaje \\
\hline $\mathrm{Si}$ & 2 & $33 \%$ \\
$\mathrm{No}$ & 4 & $67 \%$ \\
\hline Total & 6 & $100 \%$ \\
\hline
\end{tabular}


Según los resultados observados en la tabla 8 se aprecia que el $83 \%$ de los padres no saben qué hacer ante las dificultades de su hijo en tareas o en actividades de aprendizaje; por el contrario el 17\% Sí. Para la mayoría de los padres el manejo de la inatención de los hijos se hace difícil y según estos resultados no poseen estrategias que les permita saber qué hacer ante la situación. Esta característica es propia de las personas que padecen el TDAH y el impacto en la dimensión escolar es significativo por la importancia que tiene la atención en el proceso cognoscitivo para la fijación de los aprendizajes. (París, 2003).

De acuerdo a la tabla 9, el 50\% de los padres solicita información para saber qué hacer cuando su hijo no sigue instrucciones y no finaliza las tareas escolares, encargos y deberes escolares; en cambio el otro 50\% responde que sí. La tendencia es equilibrada, sin embargo es considerable el porcentaje de padres que solicitan información en esta situación. Uno de los efectos colaterales de la inatención es que el niño no sigue instrucciones o falla en terminar las cosas. DSM-IV-TR Brevario (2002).

Como se evidencia en la tabla 10, el $83 \%$ de los padres no saben qué hacer cuando su hijo(a) tiene dificultades para organizar tareas y actividades; por el contrario el otro $17 \%$ Sí sabe qué hacer. El porcentaje de los padres que no saben qué hacer ante esta eventualidad es significativo debido a que los niños con TDAH les cuesta organizarse esta situación trae como consecuencia olvidos de actividades importantes que preocupan a los representantes. Otro de los efectos colaterales causados por la inatención pues los niños tienen dificultad para organizarse. DSM-IV-TR Brevario (2002).

En la tabla 11 se puede apreciar que el $67 \%$ de los padres no sabe cómo ayudar a su hijo en cuanto a la pérdida de objetos; en cambio, el $33 \%$ sí. Se infiere que este es otro tipo de problemática que los padres no saben abordar y es consecuencia de la inatención causada por el TDAH. DSM-IV-TR Brevario (2002).

\section{DISCUSIÓN}

Según Arias (2006), dice que no basta con recolectar los datos ni con cuantificarlos adecuadamente. Una simple colección de datos no constituye una investigación. Es necesario analizarlos, compararlos y presentarlos de manera que realmente lleven a la confirmación o al rechazo de la hipótesis. Ya que en toda investigación es de importancia fundamental que los hechos y relaciones establecidas, los resultados obtenidos o los nuevos conocimientos tengan el grado máxi- 
mo de exactitud y confiabilidad. Para ello es necesario planificar una metodología ordenada que se sigue para establecer lo significativo de los hechos y fenómenos hacia los cuales se encamina el significado de la investigación.

En tal sentido, el Trastorno por Déficit de Atención y Comportamiento Perturbador (TDAH), puede ser una condición que acompañe al individuo en el transcurso de la vida, por esto se hace necesaria la intervención temprana de estas personas en el sentido de desarrollar en ellas una forma de asumirlo en procura de su bienestar.

Según Mendoza (2005), los niños y niñas con este diagnóstico deben contar con padres preparados para asumir la educación de estos con una actitud de tolerancia, paciencia e interés en el conocimiento del TDAH por estos se hace imprescindible proporcionarles respuestas efectivas y factibles en la dura tarea de formación de sus hijos que permita acompañar al maestro y a los especialistas en la atención. (p.92).

Por ello, es de vital importancia la realización del presente estudio que propone el diseño de un plan de intervención dirigido a los padres de niños con TDAH para que a través de éste se les proporcione información, estrategias necesarias que puedan aplicar y así lograr la participación efectiva en el proceso educativo de sus hijos.

Esta investigación fue factible porque se dispuso de material documental necesario para realizarla, además de contar con la colaboración de los especialistas del núcleo Psicoeducativo de la UEM Simón Rodríguez.

Asimismo, la propuesta es de gran relevancia porque se constituye en un trabajo inédito, ya que en los escritos e investigaciones consultadas no se encontró un planteamiento similar. También porque aspira aportar alternativas a la problemática proporcionando una forma de atención a los padres que requieren aprendizajes sobre el tema, lo cual fomentará la comprensión y la transformación de la realidad, y a la vez la posibilidad de obtener los elementos y estrategias que se necesitan para un mejor desarrollo de las habilidades sociales, afectivas y cognitivas de los niños que poseen trastorno por déficit de atención y comportamiento perturbador.

El beneficio de la propuesta va a repercutir en las personas directamente relacionadas con la situación, es decir, padres, familiares, maestros, compañeros; el propio plantel que contará con un recurso muy valioso aplicable en otras situaciones y por supuesto, los niños diagnosticados con TDAH. Además de constituirse en un aporte referencial a futuras investigaciones que se decidan trabajar 
sobre la temática, tal vez analizando los resultados de la aplicación del plan de intervención.

\section{PLAN DE INTERVENCIÓN}

El modelo del plan de intervención propuesto en la presente investigación se fundamenta en el modelo del Plan Educativo Individualizado (PEI) que se aplica en el aula especial una vez determinadas las necesidades y fortalezas de los sujetos objeto de estudio se procede a la planificación de actividades dirigidas a dar respuesta a la problemática planteada utilizando los recursos pedagógicos necesarios. En este sentido se encontró la siguiente definición (París 2003). "En la previsión de actividades, procedimientos, recursos y controles necesarios, para lograr los objetivos basados en el perfil de funcionamiento del sujeto objeto de atención educativa". (p. 54).

Según París (2003), todo plan se elabora partiendo de un marco teórico, filosófico y político. Dependiendo de las teorías psicológicas y pedagógicas que se manejen (p. 56). El diseño del Plan de Intervención se realizará dentro del enfoque sistémico. En este sentido, Kaufman, (1975), define sistema como “...organismo deliberadamente planeado, compuesto de elementos relacionados e interactuante, que son empleados para funcionar de manera integrada a fin de lograr propósitos predeterminados". (p. 23).

Analizando las definiciones anteriores, los propósitos serían los objetivos del Plan de Intervención, los procesos serían los pasos metodológicos a seguir y los componentes los recursos tanto humanos como materiales a seguir.

En cuanto a las etapas de planificación siguiendo el enfoque sistémico se siguió el modelo de Diseño Instruccional de Kaufman (1975), que se grafica de la siguiente manera:

1. Identificación del problema

2. Determinación de los requisitos y alternativas para la solución (Objetivos Terminales por unidad) y estos a su vez en objetivos de instrucción (Objetivos específicos) y actividad

3. Selección de estrategias de solución entre las alternativas: se refiere a "cómo hacerlo" del proceso sistémico.

4. Implantación de las estrategias de solución (Puesta en marcha).

5. Determinación de la eficacia de la realización (Control y evaluación del proceso). 
6. Revisión del sistema cuando sea necesario (Su capacidad para satisfacer las necesidades).

En este sentido, en el diseño del Plan de Intervención propuesto se cumplieron los pasos establecidos, una vez identificado el problema, se procedió a la elaboración de objetivos por unidad (generales y específicos se seleccionaron las estrategias de aplicación de esta manera se cumple con el objetivo general de la propuesta que consistió en el diseño de un Plan de intervención como respuesta a las necesidades de los padres en cuanto a la participación en el proceso educativo de sus hijos con TDAH).

\section{ELEMENTOS DEL PLAN DE INTERVENCIÓN}

- Objetivos generales y Planteados tomando en cuenta las necesidades observadas específicos en el análisis de la encuesta.

- Contenido

Información teórica actualizada del TDAH, estrategias para abordar diferentes situaciones.

- Estrategia

Basada en el modelo de instrucción de Montoya (1997):

metodológica

1. Modalidad de Instrucción.

2. Técnicas de Instrucción.

3. Organización del ambiente y grupo.

4. Organización del tiempo.

- Recursos

Humanos y materiales a emplear según los objetivos.

- Tiempo

Determinado de acuerdo a los objetivos.

Fuente: Vásquez, 2009.

\section{CONCLUSIONES}

La debilidad observada en los padres de los niños con TDAH que asisten al núcleo psicoeducativo de la Escuela Municipal Simón Rodríguez con respecto al acompañamiento eficaz del tratamiento para mejorar la vida del niño amerita el diseño del Plan de Intervención propuesto en el presente estudio con la finalidad de fomentar la participación de los padres en el proceso educativo de sus hijos con esta condición. 
Analizando los resultados se determinó que el 83\% de los padres encuestados no poseen información sobre icómo? abordar las conductas inadecuadas causadas por el TDAH de sus hijos relacionadas a la dimensión escolar, hogar y relación social. Además que el papel de la familia representada por los padres es fundamental como parte del tratamiento, ya que en el hogar las personas con TDAH desarrollan la mayor parte del tiempo de su vida y copian modelos y conductas de los jefes de familia.

Por otro lado, el aprendizaje de las personas con TDAH se encuentra severamente comprometido por la afectación de la atención que constituye uno de los elementos necesarios para el proceso mental en cuanto a la construcción del conocimiento. Es así como la hiperactividad e impulsividad causada por el $\mathrm{TDAH}$ en los niños afecta las relaciones sociales de éstos originando respuestas emocionales inadecuadas que no permite el desarrollo de ciertas habilidades sociales para las interrelaciones con sus pares, maestros y familiares.

El trastorno por déficit de atención y comportamiento perturbador afecta significativamente a la persona que lo padece en todas las áreas de la vida y origina una condición especial. Las causas no están definidas claramente; no obstante, en las últimas investigaciones se plantea que estas se derivan de factores de orden genético y se trata de un desorden fisiológico a nivel de neurotransmisores. De allí que se define como trastorno y no como enfermedad ya que hasta ahora no se ha evidenciado daño a nivel de estructura cerebral como causante del problema.

El tratamiento para el TDAH va desde la utilización de fármacos, así como terapia conductual y pedagógica que involucra especialistas como psicólogo clínico y educacional además de docentes especialistas.

\section{REFERENCIAS BIBLIOGRÁFICAS}

Ardila, A.(1992). Neuropsicología Infantil. Colombia: Prensa creativa.

Arias, F. (2006). El proyecto de Investigación. Introducción a la metodología científica. 5ae ed. Caracas: Episteme.

Kaufman, R. (1975). Planificación de sistemas educativos. México: Trillas.

Manual Diagnóstico y Estadístico de los Trastornos Mentales (DSM-IV-TR Breviario) (2002), criterios Diagnósticos. México: Masson.

Marcelli, D. (1996). Psicopatología del Niño. Barcelona: Masson. 
Mendoza, M. (2005). ¿Qué es el trastorno por déficit de atención? Una guía para padres y maestros. México: Trillas.

Montoya, A. (1997). Estrategias de Instrucción. Caracas: Fondo Editorial de la Universidad Pedagógica Experiemental Libertador.

Paris, A. (2003). Evaluación del educando con dificultades del aprendizaje. Caracas: Universidad pedagógica Experimental Libertador.

Ramírez, T. (2005). Cómo hacer un proyecto de investigación. Caracas: Panapo.

Ross, (1997). Terapia de la Conducta Infantil. México: Limusa.

Sabino, C. (1992) El Proceso de investigación. Caracas: Panapo.

Silva, J. (2006) Metodología de la Investigación. Elementos Básicos. Caracas: Co-Bo.

Tamayo, M. (1994). El proceso de la investigación científica. México: Limusa.

Van Wielink, G. (2004). Déficit de Atención con Hiperactividad. México: Trillas. 